\title{
VLT/SPHERE- and ALMA-based shape reconstruction of asteroid (3) Juno (Corrigendum)
}

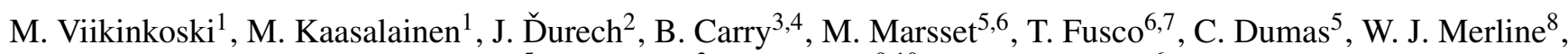 \\ B. Yang 5 , J. Berthier ${ }^{3}$, P. Kervella ${ }^{9,10}$, and P. Vernazza ${ }^{6}$ \\ 1 Department of Mathematics, Tampere University of Technology, PO Box 553, 33101 Tampere, Finland \\ e-mail: matti.viikinkoski@tut.fi \\ 2 Astronomical Institute, Faculty of Mathematics and Physics, Charles University in Prague, V Holešovičkách 2, 18000 Prague, \\ Czech Republic \\ 3 ACME, IMCCE, UMR 8028 du CNRS, UPMC, Université de Lille 1, 77 Av. Denfert-Rochereau, 75014 Paris, France \\ ${ }^{4}$ Laboratoire Lagrange, UMR 7293 CNRS, UNS, Observatoire de la Côte d'Azur, 06304 Nice, France \\ 5 European Southern Observatory (ESO), Alonso de Còrdova 3107, 1900 Casilla Vitacura, Santiago, Chile \\ 6 Aix-Marseille University, CNRS, LAM (Laboratoire d'Astrophysique de Marseille) UMR 7326, 13388 Marseille, France \\ 7 ONERA - Optics Department, 29 avenue de la Division Leclerc, 92322 Chatillon Cedex, France \\ 8 Southwest Research Institute, 1050 Walnut St., \#300 Boulder, CO 80302, USA \\ 9 Unidad Mixta Internacional FCA (UMI 3386), CNRS/INSU \& Universidad de Chile, Las Condes, Santiago, Chile \\ 10 LESIA (UMR 8109), Observatoire de Paris, CNRS, UPMC, Univ. Paris-Diderot, PSL, 5 place Jules Janssen, 92195 Meudon, \\ France
}

\section{A\&A 581, L3 (2015), DOI: 10.1051/0004-6361/201526626}

Key words. instrumentation: interferometers - instrumentation: adaptive optics - minor planets, asteroids: individual: (3) Juno methods: numerical - errata, addenda
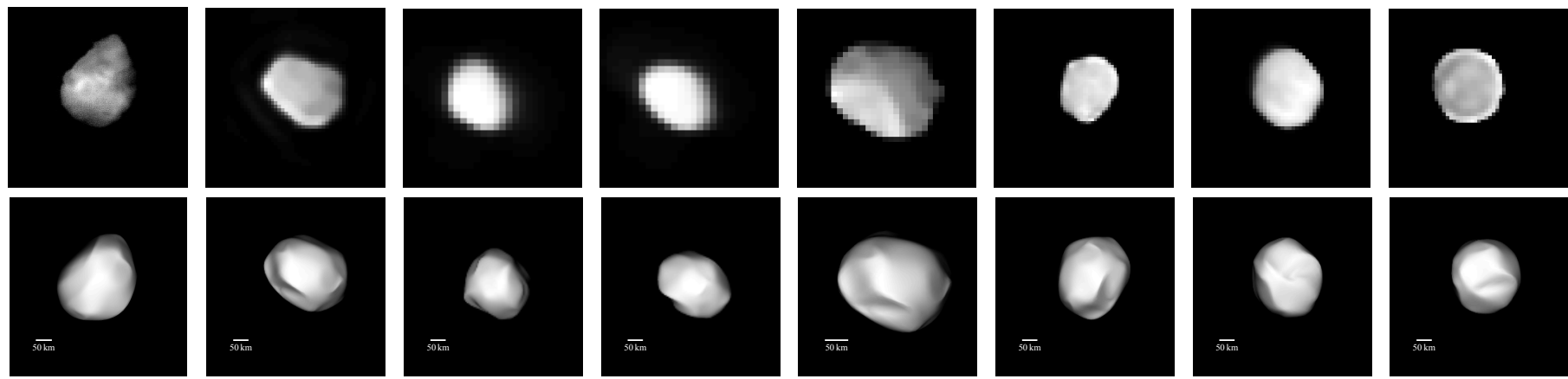

(b)

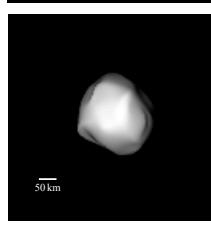

(c)

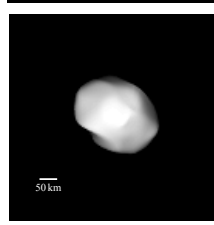

(d)

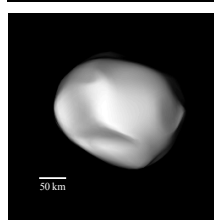

(e)

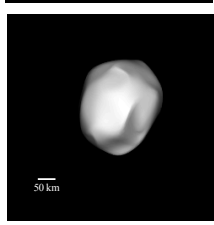

(f)

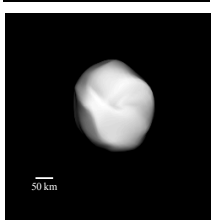

$(\mathrm{g})$

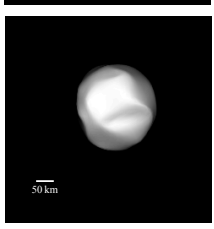

(h)

Fig. 4. Adaptive-optics images used for reconstruction (top) and corresponding model views (bottom). See Table 1 for observing conditions and instruments. The scattering law used for the shading exaggerates surface features. 